that the width of the swarm had increased very considerably since 1866.

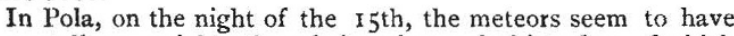
been well seen, eighty-three being observed, thirty-four of which were Leonids. The maximum of the display is stated to have taken place at $16 \mathrm{~h} .48 \mathrm{~m}$. Pola M.T., the radiant point deduced from twenty-two of the best observations being R.A. Ioh. $12 \mathrm{~m} .+26^{\circ} \cdot 5$.

\section{STUDIES OF THE LUNAR PHOTOGRAPHS TAKEN WITH THE LARGE EQUATORIAL COUDE. ${ }^{1}$}

THE new part of the "Atlas of the Moon," which we offer to the public to-day, presents many points of comparison with sections previously published. But, on account of changes of the moon's age and her libration, the portions common to both serve no double purpose. The comparison of the negatives, as we shall see more especially by studying Plates XV. and XVI., enables us to interpret some dark markings, to establish a finer distinction between the unevenness of the surface and the variations from the ordinary colour, and finally to determine more precisely the points where the reality of a periodic change may be presumed.

Plate $c$ is the third in our publication, in which the whole of the moon, visible simultaneously from the earth and sun, is represented. This portion is here much more restricted than has already appeared in Plates $a$ and $b$. The new sheet is, in consequence, less rich in detail, but derives a particularly expressive appearance, due to the rapid change from shadow to light. It is naturally the general features of the physiognomy of our satellite, the laws of distribution of plains and mountains, which can be usefully studied on these unenlarged pictures. We call special attention to the polygon form of the Mer des Crises, which is the best defined of all the dark plains of the moon; to the traces of progressive depressions which are presented in its central portions; to the large rectilinear valleys which are visible in the southern part of the disc; to the alignment following a meridian of the four most important circles of the southern horn ; to the existence of a long series of dark spots on another meridian near the limb. We find altogether, in this part of the moon, a well-marked relation between the local appearance of the surface and longitude. This relation is interesting to note, owing to the probability of its being connected with the mechanical work of tides caused by the earth.

The three unenlarged proofs published as yet demonstrate several important facts, which are confirmed by a number of clichés in our possession. They are :-

(I) A nearly continuous progression of light, extending from the terminator to the illuminated limb, approximately coincident with the curves of equal illumination and the meridians.

(2) A recrudescence of illumination in the neighbourhood of the poles, and principally of the South Pole.

(3) A marked increased of luminous intensity in the immediate neighbourhood of the limb.

A satisfactory theoretical explanation is possible for the first law, if the moon be regarded as a uniform globe in its superficial constitution, without appreciable atmosphere, beyond the state of exercising a sensible specular reflection; and reflecting indifferently in every direction the light received. Under these conditions the formula which expresses the relative intensity, and which will be found in the note relating to Plate $c$, indicates that the curves of equal illumination are the meridians, and that the intensity must increase from the terminator to the limb.

The slight exception to this rule, which is apparent near the poles, already attracted our attention when we described Plates I., II. and VI. We there saw the proof that these portions of crust very soon became solidified and rapidly acquired a great thickness. By this the polar caps have escaped the inundations originating from the interior, which have changed the appearance of the equatorial region. They are found to receive more rapidly the deposits of white cinders of the volcanic period, chief cause of the difference of tints which we observe to-day.

One may attempt to go further back, and to account for the early solidification of the polar regions. It is evident, to begin with,

1 Translation of a paper by MM. Lœwy and Puiseux. (Published by the Paris Observatory.) that the cooling must progress more quickly under the influence of a less efficient solar radiation. We will add that the tides of terrestrial origin caused smaller oscillations than in the equatorial regions, and having a slower velocity of propagation. The congealing of the superficial scoriæe is, therefore, much more easily effected near the poles.

The third fact, that is to say the abnormal increase of luminosity near the limb, merits more attention, because it is not the result of the mode of operation adopted, which, on the contrary, would be of a nature to weaken it. It shows itself in every latitude, and in all phases. In particular, the photographs taken during the partial eclipse of January 7,1898 , show that the increase of intensity on the edge is still very appreciable at opposition-that is to say, at the moment when calculation would assign a uniform brilliancy to the lunar disc.

It seems that no purely geometrical theory accounts for this appearance if it is not supposed that it is really connected with the physical state of the surface-that is to say, that not only the polar caps, but all the regions which form the apparent "contour" of the moon are, collectively, of a lighter colour than the other parts of the disc.

IIere, again, the tides of terrestrial origin, already studied from other points of view by MM. Faye and Poincaré, appear to have played an essential part. Their character is entirely modified, since the day when the rotation of the moon upon its axis was equal to the period of revolution. The periodical flow, which formerly invaded the whole equatorial region, finished by accumulating in the portion of the disc which the earth sees at the present day near its zenith. Besides this our globe, still incandescent, was really a source of great heat for its satellite. The regions near the limb have therefore entered, in their turn, into this period of low temperature and relative calm which is favourable to the consolidation of the polar regions.

The real characters of the high latitudes, already notified in the previous part, can be more completely studied here on Plates XIII. and XVII. The latter shows us the South Pole covered with mountains, varying in height from 6000 to 7000 metres, the highest that have been measured on the moon. These depths are not entirely due to the hollow of the walled plains. Although very numerous, sufficient space is left between them to allow us to judge of what was the previous contour. It can be seen that it comprised very high ridges which the craters have encroached on without destroying, and without themselves losing the regularity of their contour. There have thus been formed between the different parts of the same enclosure, differences of level which mount up to 1500 or 2000 metres. The most elevated points, which seem to correspond to a very thick crust, and capable of offering great resistance are, on the contrary, often full of little craters. The general appearance of the region gives rise to the thought, as we have already said of Plate VI., that there does not exist a covering of ice at the poles, and that it has not produced an active erosion there.

If we now consider Plate XIII. in the neighbourhood of the North Pole, we see the walled plains occupying a still more secondary place. Here the seas advance to very high latitudes. Long mountainous masses exist between them, as, for instance, the Alps and the Caucasus. These ranges, situated at a higher level than that of the seas, are strewn with summits presenting well-marked alignments, but no sign of ramified valleys, and very little circular formation. They are broken up into several frag. ments by rectilinear fissures, of which the great valley of the Alps constitutes the most celebrated and best example. The portions thus separated seem to have undergone sliding movements in relation to one another. Considerable difference of level is manifested in one massif, in such a manner that it ends at one side by a very high and very steep descent, while the other descends insensibly to the seas. The signs of primitive level have no other common feature with the terrestrial mountains than their great relative altitude, and prolonged atmospheric agencies would be necessary to make them acquire new features of resemblance with them. If the North Pole is approached, it will be seen that at the surface a net-work of furrows are formed in such a manner as to produce rectangular basins. The higher the latitude, the more important these movements of the ground become; and it is credible that, if we could see past the apparent contour of the moon, we would observe a relief com. parable with that of the South Pole. The undeniable difference which to-day exists between the appearance of the two poles is favourable to the views of G. H. Darwin and other geometers, who estimate, for reasons derived from celestial mechanics, that 
the inclination of the axis of rotation of the moon to the plane of the orbit has undergone important variations.

In order to understand the mechanism of the depressions which have caused the seas, the phenomena must be seen at different stages of advancement, and for this purpose it is necessary to study in detail how the transition between the great southern cap, which is mountainous, and the plains of the equatorial region have been effected. This passage may be studied on Plate XIV., of which nearly half, occupied by the Mer des Nuages, indicates numerous obliterated or submerged formations. The traces of the most ancient depressions have naturally disappeared by the overflowing of lava, but the more modern depressions on a dry and resisting crust, often allow their contour and size to be recognised. Some of these are connected to the central portions of the seas, leaving a great exterior band joining the mountains, which serve at the same time as limit and fulcrum. The two portions of crust thus disjointed have only been able to acquire a relative movement in a vertical direction. The part which remained immovable constitutes, in relation to the other, a sort of raised terrace. Thus must be interpreted the celebrated formation known by the name of "The Straight Wall." As the same map shows it us a little distance off, the rupture can also be accompanied by a tangential slip. It appear then like a large crevice, similar to those we saw appearing to the east of Hesiod. These fissures may be connected with large portions of the crust, may even divide chains of hills, and their form, generally rectilinear, seems independent of all the small inequalities of the surface.

It can easily be understood that such crevices rarely acquire dimensions large enough to be visible from our earth. Further, it is only near the boundaries of the seas that they have a chance of remaining open. In the central parts, the submersion of the surface has made every vestige disappear. The zones near the limb are also, by reason of the variations of the interior pressure, subject to encroachments of lava. But these inundations, which are not so frequent, are not uniformly distributed. They give rise to solid accumulations along the crevices from which they issue, and they take the form of swollen nerves. Two of these net-works can be studied in the present--and third-part; one stretching to the west of Bouillaud (Plate XIV.), the other between Landsberg and Wichmann (Plate XV.). In this last system we see a large region composed alternately of hollows and hills, as if to enable us to note the change.

The evidence of volcanic action appears here with an amplitude, a clearness, which leaves little to be desired. We have had to content ourselves with a somewhat smaller enlargemen in order to comprise in two consecutive pages (Plates XV. and XVI.) the largest portions of these brilliant aureoles, that are seen shining round certain walled plains, as Lalande, Kepler, or Copernicus. It seems to us that the comparison of these two pages is very suggestive. One is convinced by it that the diverging trails, becoming invisible by a very oblique illumination, cannot be interpreted as inequalities of the surface. Inter secting valleys and mountains without becoming fainter or deviating from their course, they cannot have been produced by subterranean or superficial means. An atmosphere agitated by variable currents seems to be the only cause which can explain the diffusion of tracks to such distances. This hypothesis further agrees with what we know of the extreme tenuity of volcanic dusts, with their capability of remaining for a long time in suspension in very thin air. It is strengthened by the fact of the existence of a relatively dark corona round the principal centres of luminosity. It is possible that in a certain zone round the more recent craters, like the region in the neighbourhood of terrestrial volcanoes, the largest projectiles, the streams of lava have got mixed in the deposits of cinders, and have not allowed them to remain clearly visible near these orifices.

Without misunderstanding the evident unity of origin of these tracks of a same system, one might be surprised to see their direction, their size, and their brightness sometimes undergoing sudden changes without clear relation to the distance of the central crater. Two disturbing causes seem to interfere: one is the meeting of high mountains, capable of dividing the atmospheric currents, of causing downfalls, and abundant condensation. The other, more frequent and more efficient, is the presence of hollow basins, still liquid at the time the downfalls of cinders took place, useless in consequence of receiving or keeping superficial deposits. The tracks, therefore, behave in the plains like a very sensitive reagent, being able to disclose by a recrudescence of brightness the smallest unevenness of the surface, and, by a sudden weakening, the slowly solidified lagoons. The comparative examination of a similar region, described in the Plates XV. and XVI. under contrary conditions, furnished numerous facts in support of this idea.

Are some of these deep basins still imperfectly dry, and will their physical state consequently be changed by a prolonged exposure to the solar rays? The green and red tints that are seen in the neighbourhood of the terminator, in the interior of some walled plains, make one think that this is so. The eye being more sensitive in the appreciation of tints, photography has the advantage of the impartial registering of relative luminous intensities. It has, without contradiction, the right to bring its evidence into the question. Plate XVII., which represents a region where the sun is setting, must be compared from this point of view with Plate I., where the sun is rising on the same parts. We find these dark spots of even tone, which in the interval have obviously modified their tints relatively to the neighbouring plateaus. The reality of this change has been confirmed by the examination of a series of clichés arranged in intermediate phases.

Below we sum up the principal ideas which this third part suggests or confirms, and which one will find developed in the following pages containing the description of the different plates. They are :-

(I) The explanation of the relative stability and the mountainous character of the polar caps.

(2) The extension of these same characters to every region which form the apparent limb.

(3) The geometric reason of the approximate coincidence that one sees between the curves of equal illumination on the disc and meridians.

(4) The origin of the abnormal recrudescence of luminosity which is shown at the apparent limb in contradiction to calculation.

(5) The difference of constitution of the two poles seems to indicate that the axis of rotation has undergone great displacements in the interior of the planet.

(6) The cause of the predominance of the seas in equatorial regions.

(7) The interpretation of the different tints that are apparent in the tracks; the use of the dark spots to recognise, amongst the sunken basins of the lunar surface, those which have been the last to solidify.

Results of equal interest can apparently be deduced from the fourth and fifth parts, the materials of which we have in hand. We hope the studious public will not have very long to wait for the rest of this work, for it has indeed been good enough to see in the two first parts an appreciable addition to our selenographic knowledge. However, we do not doubt that it is possible to do more, and also better; for if we think we have brought the methods of reproduction to the desired degree of perfection, it is not the case in the execution of direct photographs, which remains, by reason of the habitual movement of the images, a very difficult operation. A single cliché, available for enlarging, represents practically for us the only result of several months' work, and in certain phases our best proofs betray, in a very apparent way, the unsteadiness of the atmosphere.

\section{OYSTERS AND DISEASE.}

$T H I S$ research was commenced three years ago, and has been carried on intermittently in the intervals of other work.

Preliminary reports on some of our results have been laid before the British Association at the Ipswich, Liverpool, Toronto, and Bristol meetings, and a short paper on one section of the subject was communicated to the Royal Society and printed in the Proceedings last year. In the present paper we give a full account, with illustrations, of the detailed evidence upon which our various conclusions are based. The following is a brief statement of the more important results given in the paper :-

(I) Although our primary object was to study the oyster under unhealthy conditions, in order to elucidate its supposed connection with infective disease, we found it necessary to study

1 "Observations upon the Normal and Pathological Histology and Bacteriology of the Oyster." By Profs. W. A. Herdman, F.R.S., and Rubert Boyce. (Abstract of a paper read before the Royal Society, January rg.) 